\title{
ON HERMITE-FEJÉR INTERPOLATION WITH EQUIDISTANT NODES
}

\author{
G. B. BAKER, T. M. MILLS and P. VÉRTESI
}

(Received 3 April 1989)

\begin{abstract}
This paper deals with Hermite-Fejér interpolation of functions defined on a semi-infinite interval but the nodes are equally spaced. It is shown that, under certain conditions, the interpolation process has poor approximation properties.
\end{abstract}

1980 Mathematics subject classification (Amer. Math. Soc.) (1985 Revision): 41 A 05.

\section{Introduction}

Suppose that $I$ is an interval on the real line: $I$ may be open, closed or semi-open, finite or infinite. Let

$$
M=\left(x_{k, n}: k=1,2, \ldots, n ; n=1,2,3, \ldots\right)
$$

be an infinite triangular array such that

$$
x_{k, n} \in I \quad(k=1,2, \ldots, n ; n=1,2, \ldots)
$$

and, for $n=1,2, \ldots$,

$$
x_{1, n}<x_{2, n}<\cdots<x_{n, n} .
$$

For $f: I \rightarrow(-\infty, \infty)$ we define the Hermite-Fejér interpolation polynomial $H_{2 n-1}(f, x)$ to be the unique polynomial of degree $2 n-1$ or less which satisfies the $2 n$ conditions

$$
\begin{aligned}
& H_{2 n-1}\left(f, x_{k, n}\right)=f\left(x_{k, n}\right) \quad(k=1,2, \ldots, n), \\
& H_{2 n-1}^{\prime}\left(f, x_{k, n}\right)=0 \quad(k=1,2, \ldots, n) .
\end{aligned}
$$

(C) 1990 Australian Mathematical Society $0263-6115 / 90 \$ A 2.00+0.00$ 
These polynomials came into prominence in 1916 when L. Fejér [2] considered the special case where $I=[-1,1]$ and $x_{k, n}=\cos ((2 k-1) \pi /(2 n)) \quad(k=$ $1,2, \ldots, n ; n=1,2, \ldots)$. In this case we describe $M$ as being the matrix of Chebyshev nodes because, for each $n$, the points $x_{k, n}(k=1,2, \ldots, n)$ are the zeros of the Chebyshev polynomial $T_{n}(x)=\cos (n \arccos x)$. For these nodes, Fejér proved that, if $f \in C([-1,1])$, then

$$
\lim _{n \rightarrow \infty}\left\|H_{2 n-1}(f)-f\right\|=0
$$

where $\|\cdot\|$ denotes the uniform norm on the space $C([-1,1])$. Hence, Fejér proved Weierstrass' approximation theorem by using interpolation polynomials.

Since 1916, a vast literature has grown up around these polynomials: see the bibliographies in Gonska and Knoop [3] and Mills [6].

In 1958, D. L. Berman [1] investigated these polynomials where $I=$ $[-1,1]$ and the nodes were equidistant: that is

$$
x_{k, n}=-1+2(k-1) /(n-1) \quad(k=1,2, \ldots, n) .
$$

This choice of nodes is interesting because it is simple, and is the natural choice of the practical person. However, Berman shows that, in this case, the interpolation polynomials have poor approximation properties. In fact, he proves the rather remarkable fact that, if $g(t) \equiv t$, then the sequence $\left\{H_{2 n-1}(g, x): n=1,2,3, \ldots\right\}$ diverges at all points in $[-1,1] \backslash\{-1,0,1\}$. As we know that $x_{1, n}=-1$ and $x_{n, n}=1$ for all $n$, the polynomial $H_{2 n-1}(g, x)$ and the function $g(x)$ agree at these two points. We note here that some interesting convergence results concerning this choice of nodes have been stated by P. O. Runck [7].

In 1932, Szegö ([8]; see also Szegö [9, Theorem 14.7]) considered the Hermite-Fejer interpolation polynomials when the nodes of interpolation were the zeros of the Laguerre polynomial $L_{n}^{\alpha}(x)$. He proved that if $f \in$ $C([0, \infty))$ and $f(x)=O\left(x^{m}\right)$ as $x \rightarrow \infty$ for some fixed positive number $m$, then, as $n \rightarrow \infty, H_{2 n-1}(f, x) \rightarrow f(x)$ for any fixed $x \in(0, \infty)$ and the convergence is uniform in any fixed interval $[a, b]$ where $0<a<b<\infty$.

Since Szegö proved a convergence theorem which is similar to that of Fejér but set in the interval $[0, \infty)$, it is natural to ask whether there is a divergence theorem similar to that of Berman, but set in the interval $[0, \infty)$. In this paper, we will prove two such results, one in each of the following two sections. These results do not follow from applying a linear transformation to Berman's result. In the final section we will present some examples which illustrate the applicatiln of these results. Throughout this paper, $c$ or $c_{1}, c_{2}, \ldots$ denote absolute, positive constants. However, repeated use of 
any of these symbols in different expressions does not imply equality of the constants.

\section{A divergence theorem}

Before presenting our first divergence theorem, we prove the following technical lemma.

Lemma 1. Given sequences $\left\{h_{n}: n=1,2,3, \ldots\right\},\left\{j_{n}: n=1,2,3, \ldots\right\}$ and $\left\{\theta_{n}: n=1,2,3, \ldots\right\}$ such that

$$
\begin{gathered}
h_{n}>0 \quad(n=1,2,3, \ldots), \\
\lim _{n \rightarrow \infty} h_{n}=0, \quad \lim _{n \rightarrow \infty} n h_{n}=\infty, \\
\lim _{n \rightarrow \infty} j_{n} h_{n}=c>0, \\
0<c_{1}<\theta_{n}<c_{2}<1 \quad(n=1,2,3, \ldots),
\end{gathered}
$$

let

$$
K_{n}=A_{n} / B_{n}
$$

where

$$
\begin{gathered}
A_{n}=2^{2 n} h(j-1+\theta)^{2 j-3+2 \theta}(n-j+2-2 \theta)^{2 n-2 j+3-2 \theta}, \\
B_{n}=(n-2 j+2-2 \theta) n^{n-1}(n+3)^{n+2}, \\
h=h_{n}, \quad j=j_{n}, \quad \text { and } \theta=\theta_{n} .
\end{gathered}
$$

Then $\lim _{n \rightarrow \infty} K_{n}=\infty$.

Proof. From (2.1)-(2.3) it follows that

$$
\lim _{n \rightarrow \infty} j=\infty, \quad \lim _{n \rightarrow \infty}(j / n)=0 .
$$

Using (2.6) and

$$
\lim _{t \rightarrow \infty}\left(1+t^{-1}\right)^{t}=e
$$

we obtain, for $n$ sufficiently large,

$$
\begin{gathered}
(j-1+\theta)^{2 j-3+2 \theta}>c j^{2 j-3+2 \theta}, \\
(n-j+2-\theta)^{2 n-2 j+3+2 \theta}>c(n-j)^{2 n-2 j+3-2 \theta} .
\end{gathered}
$$


Thus, using (2.8), (2.9), (2.3), and (2.7) several times, we find that for values of $n$ sufficiently large,

$$
\begin{aligned}
K_{n} & >\frac{c 2^{2 n} h j^{2 j-3+2 \theta}(n-j)^{2 n-2 j+3-2 \theta}}{(n-2 j+2-2 \theta) n^{n-1}(n+3)^{n+2}} \\
& =\frac{c 2^{2 n}}{(n-2 j+2-2 \theta)}\left(\frac{h}{j}\right)\left(\frac{n-j}{n}\right)^{n-1}\left(\frac{n-j}{n+3}\right)^{n+2}\left(\frac{j}{n-j}\right)^{2 j+2 \theta-2} \\
& >\frac{c_{1} 2^{2 n} h^{2} c_{2}^{j}}{(n-2 j+2-2 \theta)}\left(\frac{j}{n-j}\right)^{2 j+2 \theta-2} \\
& >\left(c_{1} 2^{n}\right)\left(\frac{2^{n / 4}}{n-2 j+2-2 \theta}\right)\left(2^{n / 4} h^{2}\right)\left(2^{n / 4} c_{2}^{j}\right)\left(2^{n / 4}\left(\frac{j}{n-j}\right)^{2 j+2 \theta-2}\right) .
\end{aligned}
$$

Now each of these five factors increases without bound as $n$ increases. Hence $\lim _{n \rightarrow \infty} K_{n}=\infty$ and the lemma is proved.

We now state the main result of this section.

THEOREM 1. Suppose that, for $n=1,2,3, \ldots$.

$$
x_{k}=x_{k, n}=k h_{n} \quad(k=1,2, \ldots, n)
$$

where

$$
\begin{gathered}
h_{n}>0 \quad(n=1,2,3, \ldots), \\
\lim _{n \rightarrow \infty} h_{n}=0, \\
\lim _{n \rightarrow \infty} n h_{n}=\infty .
\end{gathered}
$$

Let $x$ be a positive number such that

$$
\left\{\left\{x / h_{n}\right\}: n=1,2,3, \ldots\right\} \text { has a limit point in }(0,1)
$$

and let $g(t) \equiv t$.

Then the sequence $\left\{H_{2 n-1}(g, x): n=1,2,3, \ldots\right\}$ is unbounded.

REMARK. In condition (2.14), $\left\{x / h_{n}\right\}=x / h_{n}-\left[x / h_{n}\right]$ denotes the fractional part of $x / h_{n}$.

Proof. If we define $J_{2 n-1}(g, x)$ to be the unique polynomial of degree $2 n-1$ or less such that

$$
J_{2 n-1}\left(g, x_{k}\right)=g\left(x_{k}\right) ; \quad J_{2 n-1}^{\prime}\left(g, x_{k}\right)=g^{\prime}\left(x_{k}\right) \quad(k=1,2, \ldots, n),
$$

then obviously $J_{2 n-1}(g, x) \equiv g(x)$. However, by the Hermite interpolation 
formula (see, for example, Szegö [9, (14.19)], we also have

$$
\begin{aligned}
J_{2 n-1}(g, x) & =H_{2 n-1}(g, x)+\sum_{k=1}^{n} g^{\prime}\left(x_{k}\right)\left(x-x_{k}\right) l_{k}(x)^{2} \\
& =H_{2 n-1}(g, x)+\sum_{k=1}^{n}\left(x-x_{k}\right) l_{k}(x)^{2}
\end{aligned}
$$

where

$$
\begin{gathered}
l_{k}(x)=\omega(x) /\left(\left(x-x_{k}\right) \omega^{\prime}\left(x_{k}\right)\right) \quad(k=1,2, \ldots, n), \\
\omega(x)=\left(x-x_{1}\right)\left(x-x_{2}\right) \cdots\left(x-x_{n}\right) .
\end{gathered}
$$

Hence

$$
g(x)=H_{2 n-1}(g, x)+\sum_{k=1}^{n}\left(x-x_{k}\right) l_{k}(x)^{2} .
$$

Thus, the sequence $\left\{H_{2 n-1}(g, x): n=1,2,3, \ldots\right\}$ converges (or diverges) if and only if the sequence $\left\{E_{n}(x): n=1,2,3, \ldots\right\}$ converges (or diverges) where

$$
E_{n}(x)=\sum_{k=1}^{n}\left(x-x_{k}\right) l_{k}(x)^{2} .
$$

So we focus our attention on $E_{n}(x)$.

Let $x \in(0, \infty)$ satisfy condition (2.14). Define $j \geq 1$ to be the index such that $x_{j-1} \leq x<x_{j}$ (taking $x_{0}=0$ ) and write

$$
x=(j-1) h+\theta h \quad(0 \leq \theta<1)
$$

where $h=h_{n}$. Like $h$, the variables $j, \theta$ are functions of $n$ but we will not indicate this unless it is necessary.

Using (2.18), we see that the assumption (2.14) is equivalent to assuming that the sequence $\left\{\theta_{n}: n=1,2,3, \ldots\right\}$ has a limit point in the interval $(0,1)$. So there are two constants $c_{1}, c_{2}$ such that $0<c_{1}<c_{2}<1$ and an infinite sequence of integers $n_{1}<n_{2}<n_{3}<\cdots$ such that

$$
0<c_{1}<\theta_{n}<c_{2}<1 \quad\left(n=n_{1}, n_{2}, \ldots\right) .
$$

We shall restrict $n$ to this sequence and thus makes use of (2.19) henceforth.

We now have from $(2.10),(2.15)-(2.18)$ that

$$
\omega(x)=\frac{(-1)^{n-j+1} h^{n} \Gamma(j-1+\theta) \Gamma(n-j+2-\theta)}{\Gamma(\theta) \Gamma(1-\theta)}
$$

and

$$
\omega^{\prime}\left(x_{k}\right)=(-1)^{n-k} h^{n-1} \Gamma(k) \Gamma(n-k+1) \quad(k=1,2, \ldots, n)
$$

where $\Gamma$ is the gamma function. 
From (2.15)-(2.17), (2.20)-(2.21) we obtain

$$
E_{n}(x)=G \sum_{k=1}^{n} U_{k}
$$

where

$$
G=G(n, x)=\frac{h \Gamma(j-1+\theta)^{2} \Gamma(n-j+2-\theta)^{2}}{\Gamma(\theta)^{2} \Gamma(1-\theta)^{2}},
$$

$$
U_{k}=1 /\left\{(j-k-1+\theta) \Gamma(k)^{2} \Gamma(n-k+1)^{2}\right\}, \quad k=1,2, \ldots, n .
$$

Note that

$$
\begin{aligned}
& U_{k}>0 \quad(k=1,2, \ldots, j-1), \\
& U_{k}<0 \quad(k=j, j+1, \ldots, n) .
\end{aligned}
$$

From (2.11), (2.12), (2.18) we obtain

$$
\begin{gathered}
\lim _{n \rightarrow \infty} h j=\lim _{n \rightarrow \infty} h_{n} j_{n}=x, \\
\lim _{n \rightarrow \infty} j=\infty, \\
\lim _{n \rightarrow \infty}(j / n)=0 .
\end{gathered}
$$

So, for large values of $n, j$ is much smaller than $n$. Now using (2.22) for $n$ sufficiently large,

$$
\begin{aligned}
E_{n}(x) & =G \sum_{k=1}^{n} U_{k}=G \sum_{k=1}^{j-1}\left(U_{k}+U_{n-k+1}\right)+\left(G \sum_{k=j}^{n-j} U_{k}\right) \\
& =S_{1}+S_{2}, \quad \text { say }
\end{aligned}
$$

We estimate $S_{1}$ and $S_{2}$ separately. Using (2.23), (2.24), (2.29) we find that, for $n$ sufficiently large and $1 \leq k \leq j-1$,

$$
\begin{aligned}
& \left(G U_{k}+G U_{n-k+1}\right) \Gamma(\theta)^{2} \Gamma(1-\theta)^{2} \\
& \quad=\frac{h \Gamma(j-1+\theta)^{2} \Gamma(n-j+2-\theta)^{2}}{\Gamma(k)^{2} \Gamma(n-k+1)^{2}}\left\{\frac{n-2 j+3}{(j-1-k+\theta)(n-j-k+2-\theta)}\right\} \\
& \quad>0 .
\end{aligned}
$$

Furthermore, it is not difficult to show that, when $n$ is sufficiently large, for $1 \leq k \leq(j-1)$ we have

$$
G U_{k}+G U_{n-k+1} \leq G U_{j-1}+G U_{n-j+2}
$$

Thus, from (2.30) we obtain

$$
0 \leq S_{1} \leq(j-1)\left(G U_{j-1}+G U_{n-j+2}\right) .
$$


By (2.19) we can assume that $\Gamma(\theta)=\Gamma\left(\theta_{n}\right)$ and $\Gamma(1-\theta)=\Gamma\left(1-\theta_{n}\right)$ are uniformly bounded above and below for all values of $n$. To estimate $S_{1}$, we use (2.31), (2.23), (2.24). We apply Stirling's formula [4, (8.327)]

$$
\Gamma(t)=\sqrt{(2 \pi) t^{t-(1 / 2)}} e^{-t}(1+O(1 / t)), \quad t \rightarrow \infty,
$$

and (22.7)-(2.29) to obtain

$$
S_{1}=o(1) \text { as } n \rightarrow \infty \text {. }
$$

We now assume $S_{2}$. From (2.26), (22.9) we know that if $n$ is sufficiently large, then $j<n / 2<n-j$ and hence, writing $m=[n / 2]$,

$$
-S_{2}>-G U_{m}>0 \text {. }
$$

Using (2.23), (2.24), (2.32), and Lemma 1 , we have, as $n \rightarrow \infty$,

$$
\begin{aligned}
-G & U_{m} \Gamma(\theta)^{2} \Gamma(1-\theta)^{2} \\
& =\frac{h \Gamma(j-1+\theta)^{2} \Gamma(n-j+2-\theta)^{2}}{(m-j+1-\theta) \Gamma(m)^{2} \Gamma(n-m+1)^{2}} \\
& \geq \frac{h \Gamma(j-1+\theta)^{2} \Gamma(n-j+2-\theta)^{2}}{((n / 2)-j+1-\theta) \Gamma(n / 2)^{2} \Gamma((n+3) / 2)^{2}} \\
& =c K_{n}(1+o(1)), \quad \text { where } K_{n} \text { is defined by (2.5) } \\
& \rightarrow \infty .
\end{aligned}
$$

From (3.34), (3.35) and (2.19) we have

$$
-S_{2} \rightarrow \infty \text { as } n \rightarrow \infty \text {. }
$$

The conclusion of Theorem 1 now follows from (2.30), (2.33) and (2.36).

Before stating the next result, we need a definition.

Definition. A sequence $\left\{t_{n}: n=1,2,3, \ldots\right\}$ of real numbers is uniformly distributed modulo 1 if, for every pair $a, b$ of real numbers with $0 \leq$ $a<b \leq 1$, we have $\lim _{N \rightarrow \infty} A([a, b) ; N) / N=b-a$ where $A([a, b) ; N)$ is the cardinality of the set $\left\{t_{n}: n=1,2, \ldots, N\right\} \cap[a, b)$ and $\left\{t_{n}\right\}$ is the fractional part of $t_{n}$.

The work by Kuipers and Niederreiter [5] is the standard reference on uniformly distributed sequences. We will write "u.d. mod 1 " for the phrase "uniformly distributed modulo 1 ".

Corollary 1. Assume (2.10)-(2.13). Let $x$ be a positive number such that

$$
\text { the sequence }\left\{x / h_{n}: n=1,2,3, \ldots\right\} \text { is } u . d . \bmod 1
$$

and let $g(t) \equiv t$. 
Then the sequence $\left\{H_{2 n-1}(g, x): n=1,2,3, \ldots\right\}$ is unbounded.

Proof. Since (2.14a) implies (2.14), the proof is immediate.

Theorem 1 prompts the question about what happens when $x=0$. If we consider $E_{n}(0)$ in (2.17) and use (2.15), (2.16), (2.20) with $x=0$, we find that

$$
-E_{n}(0)=\sum_{k=1}^{n} x_{k} l_{k}(0)^{2}=h \sum_{k=1}^{n} V_{k}
$$

where $V_{k}=\Gamma(n+1)^{2} /\left(k \Gamma(k)^{2} \Gamma(n-k+1)^{2}\right) \quad(k=1,2, \ldots, n)$. So, by (2.13) we find that

$$
-E_{n}(0)>h V_{n}=n h \rightarrow \infty, \quad \text { as } n \rightarrow \infty .
$$

Thus, if conditions $(2.10),(2.11),(2.13)$ hold, then the sequence $\left\{H_{2 n-1}(g, 0)\right.$ : $n=1,2,3, \ldots\}$ is unbounded.

\section{Another divergence theorem}

The second major result of this paper is the following.

THEOREM 2. Suppose that, for $n=1,2,3, \ldots$

$$
x_{k}=x_{k, n}=k h_{n} \quad(k=1,2,3, \ldots, n)
$$

where $h_{n}>0(n=1,2,3, \ldots), \lim _{n \rightarrow \infty} h_{n}=0$, and $\lim _{n \rightarrow \infty} n h_{n}=\infty$. Let $g(t) \equiv t$.

Then the sequence $\left\{H_{2 n-1}(g, x): n=1,2,3, \ldots\right\}$ diverges almost everywhere in $[0, \infty)$.

Proof. We begin by outlining the method of the proof. For $n=1,2,3$, $\ldots$, we let

$$
r_{n}=\frac{1}{\left(2 n^{3}\right)}, \quad B_{n}=\bigcup_{k=1}^{n}\left(x_{k, n}-r_{n}, x_{k, n}+r_{n}\right)
$$

and

$$
B=\left\{x: x \in B_{n} \text { for infinitely many values of } n\right\} .
$$

Denoting the Lebesgue measure of a set $X$ by $m(X)$, we note that

$$
\sum_{n=1}^{\infty} m\left(B_{n}\right)<\infty
$$

and hence, by the Borel-Cantelli lemma, $m(B)=0$. 
We will show that

$$
x \notin B \Rightarrow \limsup _{n \rightarrow \infty}\left|H_{2 n-1}(g, x)-g(x)\right|=\infty
$$

from which the conclusion of the theorem will follow immediately.

Assume that

$$
x \in[0, \infty) \backslash B .
$$

Then, by (3.1) there is an integer $n(x)$ such that if $n \geq n(x)$ then $x \notin B_{n}$. By restricting $n$ to such values, we may assume henceforth that

$$
\left|x-x_{k, n}\right| \geq r_{n} \quad(k=1,2, \ldots, n)
$$

and that, at any stage, $n$ is sufficiently large for our purposes.

We now follow the argument employed to prove Theorem 1, step by step, with some minor modifications. The proof is unchanged up to and including (2.18). To replace (2.19), observe that (3.4) implies that

$$
1 /\left(2 n^{3} h\right) \leq \theta \leq\left(2 n^{3}-1\right) /\left(2 n^{3} h\right) \text {. }
$$

We can use the identity

$$
\Gamma(\theta) \Gamma(1-\theta)=\pi /(\sin \pi \theta) \quad(0<\theta<1)
$$

[4, formula $8.334(3)]$ and replace $(2.19)$ by

$$
\pi \leq \Gamma(\theta) \Gamma(1-\theta) \leq n^{3} h .
$$

The argument from (2.20)-(2.31) can be used without change. To estimate $S_{1}$, use the left-hand inequality of (3.5) and obtain (2.33). To estimate $S_{2}$, use (2.34) and the right-hand inequality in (3.5). We then obtain, instead of (2.35),

$$
-G U_{m}>K_{n}(1+o(1)) /\left(n^{3} h\right), \quad \text { as } n \rightarrow \infty .
$$

We now use the proof of Lemma 1 without any change until the last line to show that, as $n \rightarrow \infty$,

$$
K_{n} /\left(n^{3} h\right) \rightarrow \infty
$$

and this gives us (2.36). Thus, assuming (3.3) we have

$$
\limsup _{n \rightarrow \infty}\left|H_{2 n-1}(g, x)-g(x)\right|=\infty
$$

and so Theorem 2 is established.

\section{Examples}

It is interesting to compare Theorem 1 with Theorem 2 . Theorem 1 provides us with a way of determining if the sequence

$$
S(x)=\left\{H_{2 n-1}(g, x): n=1,2,3, \ldots\right\}
$$


diverges for a given value of $x$ but it does not giv us any of how many such values there may be. Theorem 2 tells us that $S(x)$ diverges almost everywhere in $[0, \infty)$ but it does not tell us for which values $x$ the sequence diverges. Between the two results, we can often manage to sort out many particular situations. We now present some examples to show how th theorems may be used.

EXAMPle 1. Consider the case when $H_{n}=1 /(\sqrt{(} a n+b)$ where $a>0$, $b$ are constants. It is clear that the only difficult in applying Theorem 1 is checking (2.14). Often, checking (2.14a) in Corollary 1 is easier: this can be done by referring to results in Kuipers and Niederreiter [5]. In this example, we find from [5, Corollary 2.1] that, for all $x>0,(2.14 \mathrm{a})$ is satisfied and hence $S(x)$ diverges for all $x>0$. Thus, by the remarks at the end of Section 2, $S(x)$ diverges for all $x \geq 0$. In passing, we note that in Berman's result, the divergence did not occur at the point $x=0$ in the interval $[-1,1]$.

EXAMPle 2. Consider the case when $h_{n}=1 /(\log n), n>1$. From [5, Theorem 2.6] we find that, for all $x>0$, condition (2.14a) is not satisfied. In this case, while Theorem 2 assures us that $S(x)$ diverges for almost all $x>0$, Corollary 1 does not help us to identify any such value of $x$. However, by using another argument from [5, Example 2.5] we can show that for $x>0$ the sequence $\{\{x \log n\}: n=2,3, \ldots\}$ is dense in $[0,1]$ and hence has a limit point in $(0,1)$. Thus, $S(x)$ diverges for all $x>0$ (and hence all $x \geq 0)$.

EXAMPLE 3. Consider the case when $h_{n}=1 /[\sqrt{n}]$ where $[t]$ is the integer part of $t$. If $m$ is a natural number, then, for $n$ sufficiently large, we can find $k \in\{1,2, \ldots, n\}$ such that $m=k h_{n}$ and hence $H_{2 n-1}(g, m)=g(m)$. Thus $S(x)$ converges whenever $x$ is a natural number. From this example we see that in general, the "a.e. divergence" in Theorem 2 cannot be replaced by "everywhere divergence".

Example 4. Consider the case when $h_{n}=1 \quad(n=1,2,3, \ldots)$. This is not covered by any results in this paper, but it is worth mentioning that, in this case $S(x)$ diverges for all values of $x \geq 0$ unless $x$ is a natural number and hence, ultimately a node of interpolation. Modifying the proof of Theorem 1 will show this.

\section{Acknowledgements}

T. M. Mills acknowledges financial support from Bendigo College of Advanced Education and the Hungarian Ministry for Education and Culture. 
P. Vértesi acknowledges financial support from the Hungarian National Foundation for Scientific Research (Grant No. 1801).

\section{References}

[1] D. L. Berman, 'Divergence of the Hermite-Fejér interpolation process' (Russian), Uspehi Mat. Nauk 13 (80) (1958), no. 3, 143-148.

[2] L. Fejér, 'Über Interpolation', Göttinger Nachrichten 1 (1916), 66-91.

[3] H. H. Gonska and H. B. Knoop, 'On Hermite-Fejér interpolation: a bibliography (19141987)', Studia Sci. Math. Hungar, to appear.

[4] I. S. Gradshteyn and I. M. Ryzhik, Tables of integrals, series, and products, (Corrected and enlarged edition, Academic Press New York, 1980).

[5] L. Kuipers and H. Niederreiter, Uniform distribution of sequences (Wiley, New York, 1974).

[6] T. M. Mills, 'Some techniques in approximation theory', Math. Sci. 5 (1980), 105-120.

[7] Paul Otto Runck, 'Sur la convergence des polynomes d'interpolation de Lagrange et d'Hermite aux noeuds equidistants' C. R. Acad. Sci. Paris Sér. II Méc. Phys. Chim. Sci. Univers Sci. Terre 245 (1957), 1211-1213.

[8] G. Szegö, 'Über gewisse Interpolationspolynome, die zu den Jacobischen und Laguerreschen Abszissen gehören', Math. Z. 35 (1932), 579-602.

[9] Gábor Szegö, Orthogonal polynomials (4th ed., Colloq. Publ. 23, Amer. Math. Soc., Providence, R. I., 1975).

Towers, Perrin, Forster and Crosby

356 Collins Street

Melbourne

Victoria 3000

Australia

Mathematical Institute

Hungarian Academy of Sciences

P. O. Box 127

Budapest 1364

Hungary
Bendigo College of Advanced

Education

P. O. Box 199, Bendigo

Victoria 3550

Australia 